\title{
Abundância e riqueza de espécies de euglossini (hymenoptera: apidae) em diferentes fragmentos no município de Mojuí dos Campos, Pará, Brasil
}

As abelhas da tribo Euglossini (Hymenoptera: Apidae) são extremamente importantes na polinização das florestas neotropicais e por serem sensíveis a mudanças em seu habitat, são consideradas excelentes bioindicadores de qualidade ambiental. Nesse contexto, o presente estudo objetivou (i) comparar a riqueza de espécies e abundância de indivíduos de Euglossini entre fragmento de floresta, pastagem e plantação de soja; (ii) identificar a preferência pelas iscas odoríferas; e (iii) avaliar o efeito de borda sobre a riqueza de espécies e abundância de indivíduos. O estudo foi realizado no município de Mojuí dos Campos, Pará, Brasil, em três áreas: fragmento de floresta, pastagem e plantação de soja. Utilizou-se seis iscas odoriferas para atrair os machos: eugenol, eucaliptol, vanilina, benzoato de benzila, cinamato de metila e geraniol. Foram coletados 1668 indivíduos distribuídos em quatro gêneros e 11 espécies. Existe diferença significativa na abundância de Euglossini entre os ambientes $(F(2,24)=4,1007 ; p=0,029)$, a maior abundância ocorreu na área de pastagem $(866-52 \%)$. Houve diferença significativa diferença significativa na abundância de Euglossini entre os ambientes $(F(2,24)=4,1007 ; p=0,029)$, a maior abundância ocorreu na área de pastagem $(866-52 \%)$. Houve diferença significativa
tanto na abundância $(F(5,156)=15,811 ; p<0,001)$ quanto na riqueza de espécies $(F(5,156)=70,039 ; p<0,001)$ de Euglossini entre as iscas. Vanilina mostrou-se a isca mais atrativa e geraniol a menos atrativa. Não há diferença significativa na abundância $(F(2,24)=1,8505 ; p=0,178)$ e riqueza de espécies de Euglossini $(F(2,24)=0,299 ; p=0,744)$ entre os transectos borda, intermediário e centro, o que pode estar relacionado com a proximidade entre as áreas e a capacidade das abelhas de voar longas distâncias. Outro fator que pode influenciar nesse resultado, é a capacidade de dispersão dessas abelhas, que possibilita a exploraração localidades próximas. Fatores climáticos, diferença na volatilidade das substâncias, faixa etária das populações, pureza e composição isométricas das substâncias e ofertas de fontes naturais podem ter influenciado nesse resultado. Apesar dos resultados mostrarem um alto grau de degradação na áre estudada, é necessário a realização de mais estudos, que levem em conta o tamanho dos fragmentos e o número de Eulaema nigrita em relação a outras espécies para que se possa obter dados mais precisos sobre os impactos dos diferentes usos da terra na diversidade das abelhas Euglossini.

Palavras-chave: Euglossini; Usos da terra; Alteração ambiental; Preferência por iscas odoríferas.

\section{Abundance and species richness of euglossini (hymenoptera: apidae) in different fragments in the municipality of Mojuí dos Campos, Pará, Brazil}

\begin{abstract}
The bees of the Euglossini tribe (Hymenoptera: Apidae) are extremely important in the pollination of Neotropical forests and because they are sensitive to changes in their habitat, they are considered excellent bioindicators of environmental quality. In this context, the present study aimed to (i) to compare the species richness and abundance of Euglossini between fragments of forest, pasture and soy plantation; (ii) identify the preference for chemical baits; and (iii) assess the edge effect on species richness and abundance of individuals. The study was carried out in the municipality of Mojui dos Campos, Pará, Brazil, in three distinct fragments: forest fragment, pasture and soybean plantation. Six chemical baits were used to attract males: eugenol, eucalyptol, vanillin, benzyl benzoate, methyl cinnamate and geraniol. A total of 1668 individuals were collected in 4 genera and 11 species. There is a significant difference in the abundance of Euglossini between the environments $(F(2,24)=4,1007 ; p=0.029)$, the highest abundance occurred in the pasture area $(866-52 \%)$. There was a significant difference in both abundance $(F(5,156)=15.811 ; p<0.001)$ and in the species richness $(F(5,156)=70.039 ; p<0.001)$ of Euglossini among the baits. Vanillin proved to be the most attractive bait and geraniol the least $(F(5,156)=15.811 ; p<0.001)$ and in the species richness $(F(5,156)=70.039 ; p<0.001)$ of Euglossini among the baits. Vanillin proved to be the most attractive bait and geraniol the least
attractive. There is no significant difference in abundance $(F(2,24)=1,8505 ; p=0.178)$ and Euglossini species richness $(F(2,24)=0.299 ; p=0.744)$ between the border, intermediate and center transects, which may be related to the proximity between the areas and the bees' ability to fly long distances. Another factor that can influence this result is the dispersal capacity of these bees, and they can explore nearby localities. Climatic factors, difference in substance volatility, age range of populations, isometric purity and composition of substances and supplie from natural sources may have influenced this outcome. Although the results show a high degree of degradation in the studied area, it is necessary to carry out further studies, taking into account the size of the fragments and the number of Eulaema nigrita in relation to other species in order to obtain more accurate data on the impacts of different soil uses on the diversity of Euglossini bees.
\end{abstract}

Keywords: Euglossini; Land uses; Environmental change; Preference for chemical baits.

Debro Acácio Jonatien Batista (iD) Universidade Federal do Oeste do Pará, Brasil http://lattes.cnpq.br/5791749210546389 http://orcid.org/0000-0003-1060-2227 debro.acacio@gmail.com

Fagner Araújo Santos (iD)

Universidade Federal do Oeste do Pará, Brasil http://lattes.cnpq.br/1713983297933074 http://orcid.org/0000-0003-3303-1279 fagnerdantona@gmail.com

Gleicy Assunção Rodrigues (iD

Universidade Federal do Oeste do Pará, Brasil http://lattes.cnpq.br/3768457549987541 http://orcid.org/0000-0001-5958-7000 gleicyrodrigues455@gmail.com

DOI: 10.6008/CBPC2179-6858.2020.005.0018

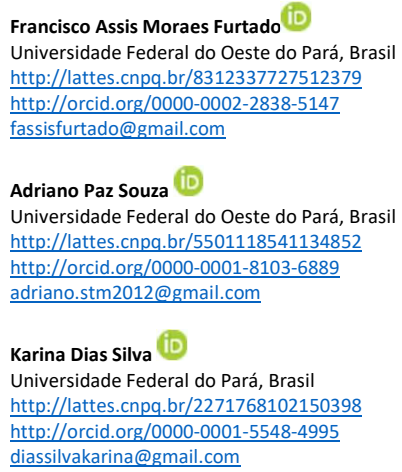

DOI: 10.6008/CBPC2179-6858.2020.005.0018

\section{Referencing this:}

BATISTA, D. A. J.; SANTOS, F. A.; RODRIGUES, G. A.; FURTADO, F. A. M.; SOUZA, A. P.; SILVA, K. D.; OLIVEIRA JUNIOR, J. M. B.. Abundância e riqueza de espécies de euglossini (hymenoptera: apidae) em diferentes fragmentos no município de Mojuí dos Campos, Pará, Brasil. Revista Ibero Americana de Ciências Ambientais, v.11, n.5, p.174-185, 2020. DOI: http://doi.org/10.6008/CBPC2179- 


\section{INTRODUÇÃO}

A expansão do agronegócio na Amazônia traz como consequência um alto e acelerado índice de desmatamento, em grande parte na região oeste do Pará, que tem como as principais causas a pecuária e agricultura de larga escala (DEFRIES et al., 2002). Nas áreas rurais dos municípios de Santarém, Mojuí dos Campos e Belterra (Pará, Brasil), as paisagens encontram-se fragmentadas, a monocultura de soja e pastagem vem substituindo grande parte da vegetação nativa, podendo afetar diretamente a biodiversidade, inclusive abelhas da tribo Euglossini (Hymenoptera: Apidae), importantes polinizadoras de muitas espécies botânicas (DRESSLER, 1968).

Por serem sensíveis às mudanças em seu habitat, essas abelhas são consideradas potenciais bioindicadores de qualidade do habitat (RAMALHO et al., 2009). Na Mata Atlântica, já foram comprovadas evidências dos impactos da fragmentação na população dessas abelhas (NEMÉSIO et al., 2007; 2010). Na Amazônia, principalmente no oeste do Pará, são escassas informações sobre os efeitos da retirada da cobertura vegetal na população de Euglossini.

Euglossini é uma das quatro tribos de Apidae corbiculadas, popularmente conhecidas como abelhas das orquídeas, possuem as tíbias posteriores dilatadas, lisas e côncavas na parte externa para o transporte de pólen e materiais para construção de ninhos (MICHENER, 2000). Está dividida em cinco gêneros - Euglossa Latreille, 1802; Eulaema Lepeletier, 1841; Eufriesea Cockerell, 1908; Exaerete Hoffmannsegg, 1817; Aglae Lepeletier \& Serville, 1825 - com 200 espécies reconhecidas (ROUBIK et al., 2004). As abelhas Euglossini são solitárias, de voo rápido e coloração geralmente metálica. Pertencem exclusivamente a região Neotropical e estão distribuídas desde o sul dos Estados Unidos até o norte da Argentina (MINCKLEY et al., 1996).

As Euglossini apresentam alta diversidade em florestas úmidas (ROUBIK, 2004), no Brasil são descritas cerca de 57 espécies para Mata Atlântica (PERUQUETTI et al., 1999). Para a Floresta Amazônica o número de espécies conhecidas é de 83 (REBÊLO, 2001). Em regiões mais abertas como Cerrado e Caatinga, poucas espécies foram descritas (DRESSLER, 1982; ZANELLA, 2000).

Flores que necessitam de vibração para liberação de pólen como as de Cassia (Leguminosae), Sauvagesia (Ochenaceae), Solanum (solanaceae) e Tibouchina (Melastomataceae), juntamente com outra variedade de espécies florais de diversos gêneros e famílias distintas são fontes de pólen para Euglossini (BEZERRA et al., 2003; DRESSLER, 1982). Portanto, ao buscarem essa variedade de recursos florais, essas abelhas se tornam potenciais polinizadores de cerca de 30 famílias de plantas, incluindo mais de 2000 espécies de orquídeas (CAMERON, 2004; CARVALHO et al., 2002; DODSON et al., 1969; DRESSLER, 1968, 1982; MARTINI et al., 2003).

A utilização de iscas odores para a atração e coleta de machos é uma técnica amplamente conhecida (REBÊLO, 2001). Os machos de Euglossini são facilmente atraídos por certos terpenoides e hidrocarbonetos aromáticos sintéticos, análogos àqueles presentes nas fragrâncias florais (HILLS et al., 1972). Os machos são normalmente velozes e ariscos, porém, certas substâncias os deixam lentos e embriagados facilitando a sua coleta (DODSON et al., 1969). As iscas odoríferas mais comumente utilizadas são eugenol, vanilina, cineol, 
eucaliptol e cinamato de metila. Há diversos trabalhos que mostram as iscas odoríferas preferidas pelas diversas espécies de Euglossini, no entanto, ainda não há uma padronização com relação aquelas que trariam melhores resultados caso fossem empregadas (FARIAS et al., 2013).

Alguns trabalhos têm investigado os impactos do desmatamento e da fragmentação de florestas na população dessas abelhas e os resultados têm mostrado que o desmatamento tem um efeito negativo na população de algumas espécies de Euglossini (MORATO, 1994; SOFIA et al., 2004; TONHASCA JUNIOR et al., 2003). Essas abelhas são capazes de voar longas distâncias em florestas contínuas, e podem transpor fragmentos florestais separados por 1500 metros (JAZEN, 1971; TONHASCA JUNIOR et al., 2003). No entanto, estudos revelam que áreas desmatadas, como as que estão surgindo na área rural dos municípios de Santarém e Mojuí dos Campos, podem constituir barreiras para determinadas espécies, comprometendo sua variabilidade genética (MORATO, 1994; PERUQUETTI et al., 1999). As Euglossini são consideradas espécieschave, portanto, têm uma influência acentuada no caráter ou estrutura de um ecossistema, podendo desencadear um efeito cascata atingindo vários organismos do ecossistema caso sejam removidas ou tenham suas atividades interrompidas (LASALLE et al., 1993). A conservação desses importantes visitantes florais é fundamental para a manutenção da estabilidade das comunidades vegetais onde eles são encontrados (POWELL et al., 1987).

Nesse contexto, o presente estudo teve como objetivos (i) comparar a riqueza de espécies e abundância de indivíduos de Euglossini entre fragmentos de floresta, pastagem e plantação de soja; (ii) identificar a preferência pelas iscas odoríferas; e (iii) avaliar o efeito de borda sobre a riqueza de espécies e abundância de indivíduos.

\section{MATERIAIS E MÉTODOS}

\section{Área de estudo}

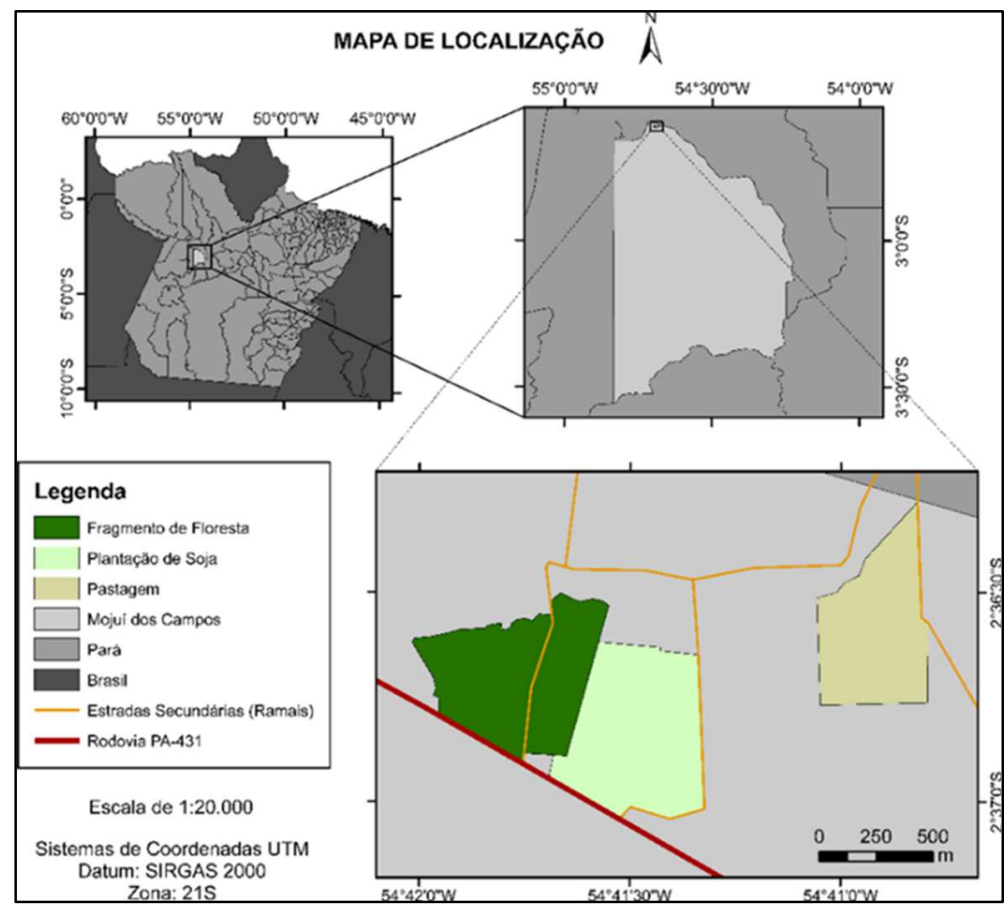

Figura 1: Mapa de localização das três áreas estudadas no município de Mojuí dos Campos, Pará, Brasil. 


\section{Coleta de dados}

O estudo foi desenvolvido em três áreas: fragmento de floresta, pastagem e plantação de soja, no município de Mojuí dos Campos, localizado na PA- 431 a 32 km de Santarém (floresta - S02 $36.635^{\prime}$ e W54 $4^{\circ} 41.588^{\prime}$; pastagem - S02 ${ }^{\circ} 36.320^{\prime}$ e W54 $4^{\circ} 40.854^{\prime}$; soja - S02 ${ }^{\circ} 36.632$ e W54 ${ }^{\circ} 41.576$ ') estado do Pará, Brasil (Figura 1). O clima dominante na região é quente e úmido, característico das Florestas Tropicais, e não está sujeito a elevadas mudanças de temperatura devido sua proximidade da Linha do Equador, variando anualmente entre $25 \circ$ a $28 \circ \mathrm{C}$, com a umidade relativa média do ar de $86 \%$ e a precipitação média anual é de $1920 \mathrm{~mm}$ (IBGE, 2015).

Foram utilizadas armadilhas confeccionadas de garrafas plásticas tipo PET de dois litros (NEVES et al., 1997), com duas aberturas laterais, contendo um chumaço de algodão envolvido por gaze, pendurado em um barbante de modo que as aberturas laterais ficassem alinhadas com o chumaço (Figura 2).

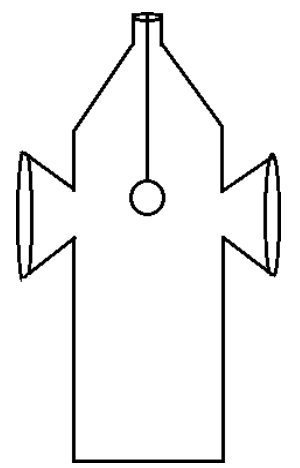

Figura 2: Armadilha confeccionada a partir de garrafa PET descartável de dois litros utilizada para capturar os machos Euglossini nas três áreas de estudo no município de Mojuí dos Campos, Pará, Brasil.

As armadilhas foram distribuídas em três conjuntos a 200 metros um do outro, em cada área de estudo (fragmento de floresta, pastagem e plantação de soja). Cada conjunto era composto por três fileiras paralelas separadas por 40 metros (borda, intermediário e centro do fragmento), e cada fila com seis armadilhas distantes cinco metros uma da outra, penduradas em estacas de madeira nas áreas de pastagem e plantação de soja, e nos troncos das árvores na área de fragmento de floresta, a uma altura de 1,70m do solo, totalizando 56 armadilhas por área (Figura 3). Para atrair as abelhas, em cada fila foram utilizadas seis iscas odoríferas distintas na seguinte ordem: eugenol, eucaliptol, vanilina, benzoato de benzila, cinamato de metila e geraniol (Figura 3). Foram colocadas três gotas nos chumaços de algodão, seguindo o método descrito por Neves et al. (1999).

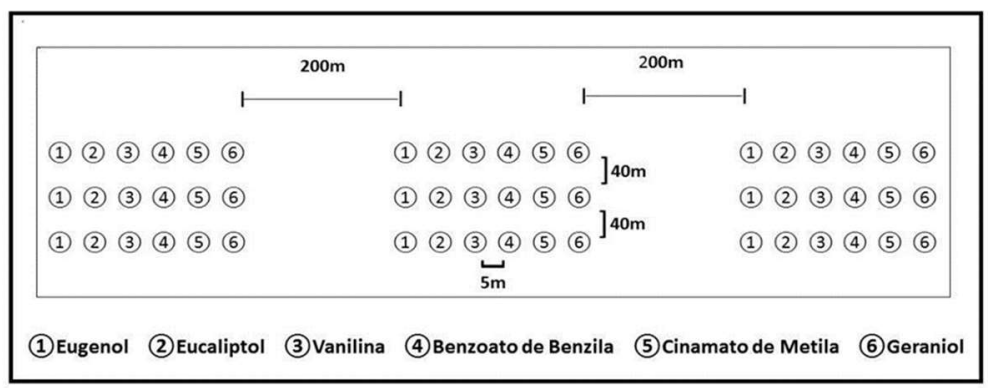

Figura 3: Representação da distribuição padrão das armadilhas nas áreas de fragmento de floresta, pastagem e plantação de soja no município de Mojuí dos Campos, Pará, Brasil. 
Foram realizadas 15 coletas em cada área, no período entre 23 de setembro e 22 de novembro de 2016. As iscas odoríferas foram colocadas em gotas nos chumaços de algodão dentro das armadilhas no período entre as 16:00hs e 17:00hs, e no mesmo horário do dia seguinte as abelhas capturadas eram retiradas e acrescentava-se mais três gotas de isca odorífera para manter as iscas com o mesmo potencial atrativo.

Os Euglossini capturados foram conservados em álcool 70\% e identificados em laboratório com uso de estereomicroscópios, com auxílio de chaves taxonômicas especializadas (ANJOS-SILVA et al., 2006; KIMSEY, 1982; MOURE, 2000; OLIVEIRA, 2006). Após a identificação os espécimes foram depositados como material testemunho no Laboratório Multidisciplinar de Gestão Ambiental da Universidade Federal do Oeste do Pará, Pará, Brasil.

\section{Análise dos dados}

Para comparar a riqueza de espécies e abundância de indivíduos entre áreas de pastagem, plantação de soja e fragmento de florestal foi utilizada a Análise de Variância de um critério (ANOVA one-way), adotando os dados de abundância e riqueza por amostra e por ambiente (AYRES et al., 2007; ZAR, 1999). A ANOVA também foi utilizada para identificar a preferência das abelhas Euglossini pelas iscas odoríferas utilizadas e para testar o efeito de borda. Foram testados os pressupostos de normalidade (Shapiro-Wilk) e homocedasticidade (teste de Levene) (ZAR, 2010) Todas as análises foram realizadas pelas rotinas do programa $R$ (R DEVELOPMENT CORE TEAM, 2011), utilizando o pacote vegan.

\section{RESULTADOS}

\section{Descrição da comunidade de Euglossini}

Foram amostrados 1.668 indivíduos de Euglossini, distribuídos em quatro gêneros e 11 espécies (Figura 4; Tabela 1). Ambiente de pastagem apresentou o maior de número de indivíduos ( $N=866 ; 52 \%$ ), seguido de ambiente de plantação de soja ( $N=456 ; 27 \%$ ) e fragmento de floresta ( $N=346 ; 21 \%$ ) (Figura 3; Tabela 1).

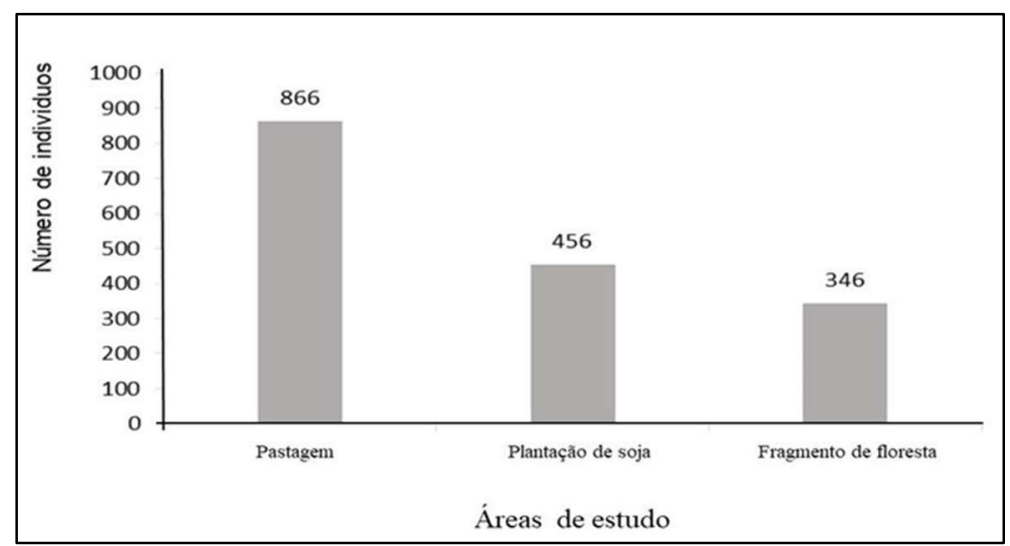

Figura 4: Número de indivíduos de Euglossini coletados nos ambientes de fragmento de floresta, pastagem e plantação de soja no município de Mojuí dos Campos, Pará, Brasil. 
Tabela 1: Números de machos coletados nos três fragmentos estudados na região de Mojuí dos Campos, Pará, Brasil, no período de 23 de setembro e 22 de novembro de 2016.

\begin{tabular}{|c|c|c|c|c|c|}
\hline \multirow[t]{2}{*}{ Espécies } & \multicolumn{2}{|c|}{ Ambientes } & \multirow[b]{2}{*}{ Soja } & \multirow[t]{2}{*}{ Total } & \multirow[t]{2}{*}{$\%$} \\
\hline & Floresta & Pastagem & & & \\
\hline Euglossa allosticta (Moure, 1969) & 4 & 1 & 0 & 5 & 0 \\
\hline Eulaema bombiformis (Packard, 1869) & 16 & 12 & 4 & 32 & 2 \\
\hline Eulaema bomboides (Friese, 1923) & 55 & 53 & 44 & 152 & 9 \\
\hline Euglosa imperialis Cockerell, 1922 & 2 & 6 & 6 & 14 & 1 \\
\hline Euglossa iopyrrha (Dressler, 1982) & 4 & 7 & 3 & 14 & 1 \\
\hline Eulaema mocsaryi (Friese, 1899) & 127 & 131 & 78 & 336 & 20 \\
\hline Eulaema nigrita (Lepeletier, 1841) & 58 & 532 & 267 & 857 & 51 \\
\hline Eufriesea ornata (Mocsáry, 1896) & 1 & 0 & 0 & 1 & 0 \\
\hline Exaerete smaragdina (Guérin-Méneville, 1845) & 7 & 7 & 3 & 17 & 1 \\
\hline Eufriesea surinamensis (Linnaeus, 1758) & 4 & 1 & 2 & 7 & 4 \\
\hline Euglossa truncata (Rebêlo et al., 1995) & 68 & 116 & 49 & 233 & 14 \\
\hline Total & 346 & 866 & 456 & 1668 & 100 \\
\hline$\%$ & 21 & 52 & 27 & 100 & \\
\hline
\end{tabular}

\section{Abundância e riqueza de espécies em distintos fragmentos}

Existe diferença significativa da abundância de Euglossini entre os ambientes $\left(\mathrm{F}_{(2,24)}=4,101 ; p=0,029\right)$ (Figura 5). 0 teste Tukey mostrou que ambiente de pastagem apresenta em média 46 indivíduos a mais do que ambientes de plantação de soja, e em média 58 indivíduos a mais do que fragmentos de floresta. Ambientes de plantação de soja não diferem de fragmentos de floresta em termo de abundância. Não foi observada diferença significativa na riqueza de espécies entre as áreas estudadas $\left(F_{(2,24)}=3,070 ; p=0,065\right)$.

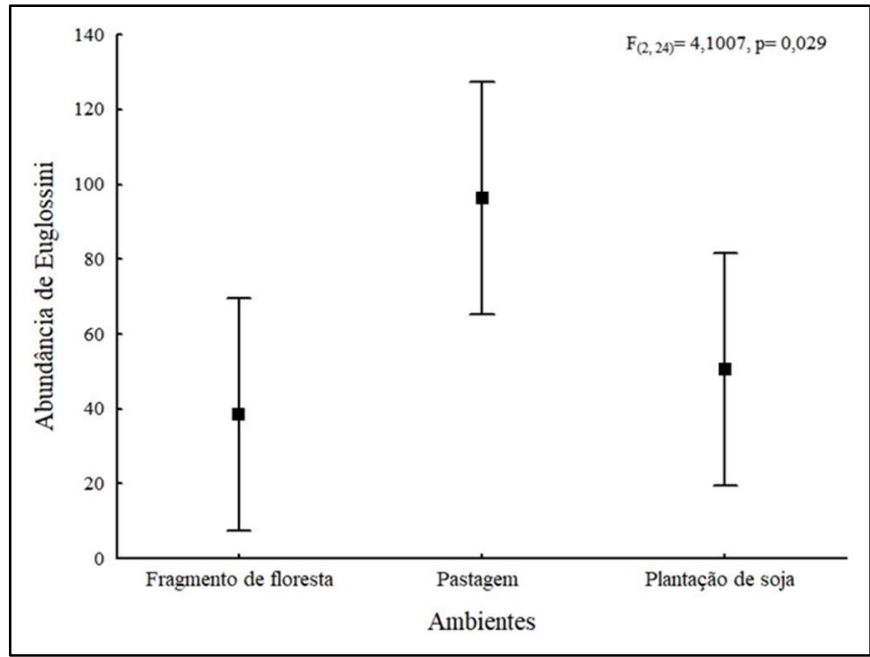

Figura 5: Comparação da abundância de espécies de Euglossini entre os fragmentos estudados (fragmento de floresta, pastagem e plantação de soja) no município de Mojuí dos Campos, Pará, Brasil.

\section{Preferência por iscas odoríferas}

Vanilina mostrou-se a isca mais atrativa $(61,03 \%)$, seguida por Eugenol $(29,79 \%)$. Geraniol atraiu apenas $0,23 \%$, sendo a isca com menor potencial atrativo (Tabela 2 ).

Tabela 2: Número de machos de Euglossini atraídos por iscas odoríferas entre os fragmentos estudados (fragmento de floresta, pastagem e plantação de soja) no município de Mojuí dos Campos, Pará, Brasil. Legenda: Eug - Eugenol; Euc Eucaliptol; Van - Vanilina; Ben - Benzoato de Benzila; Cin - Cinamato de Metila; Ger - Geraniol.

\begin{tabular}{|c|c|c|c|c|c|c|c|}
\hline \multirow{2}{*}{ Espécies } & \multicolumn{6}{|c|}{ Iscas odoríferas } & \multirow{2}{*}{ Total } \\
\hline & Eug & Euc & Van & Ben & Cin & Ger & \\
\hline Euglossa allosticta (Moure, 1969) & 3 & 0 & 1 & 1 & 0 & 0 & 5 \\
\hline Eulaema bombiformis (Packard, 1869) & 7 & 0 & 11 & 0 & 14 & 0 & 32 \\
\hline Eulaema bomboides (Friese, 1923) & 110 & 3 & 37 & 1 & 1 & 0 & 152 \\
\hline
\end{tabular}


Euglosa imperialis Cockerell, 1922

Euglossa iopyrrha (Dressler, 1982)

Eulaema mocsaryi (Friese, 1899)

Eulaema nigrita (Lepeletier, 1841)

Eufriesea ornata (Mocsáry, 1896)

Exaerete smaragdina (Guérin-Méneville, 1845)

Eufriesea surinamensis (Linnaeus, 1758)

Euglossa truncata (Rebêlo et al., 1995)

Total

$\%$

Houve diferença significativa na abundância de Euglossini entre as iscas odoríferas $\left(F_{(5,156)}=15,811\right.$; $p<0,001$ ) (Figura 6A). O teste Tukey mostrou que vanilina foi a isca com maior preferência, atraindo em média 19 indivíduos a mais do que eugenol, 36 a mais que geraniol, 35 a mais que eucaliptol, 34 a mais que cinamato de metila e 37 a mais que benzoato de benzila. 0 teste Tukey também mostrou que eugenol foi a segunda isca mais atrativa, atraindo 18 indivíduos a mais do que geraniol, 17 a mais que eucaliptol, 15 a mais que cinamato de metila e 18 a mais que benzoato de benzila. As iscas geraniol, eucaliptol, cinamato de metila e benzoato de benzila não apresentaram diferença significativa em termos de abundância.
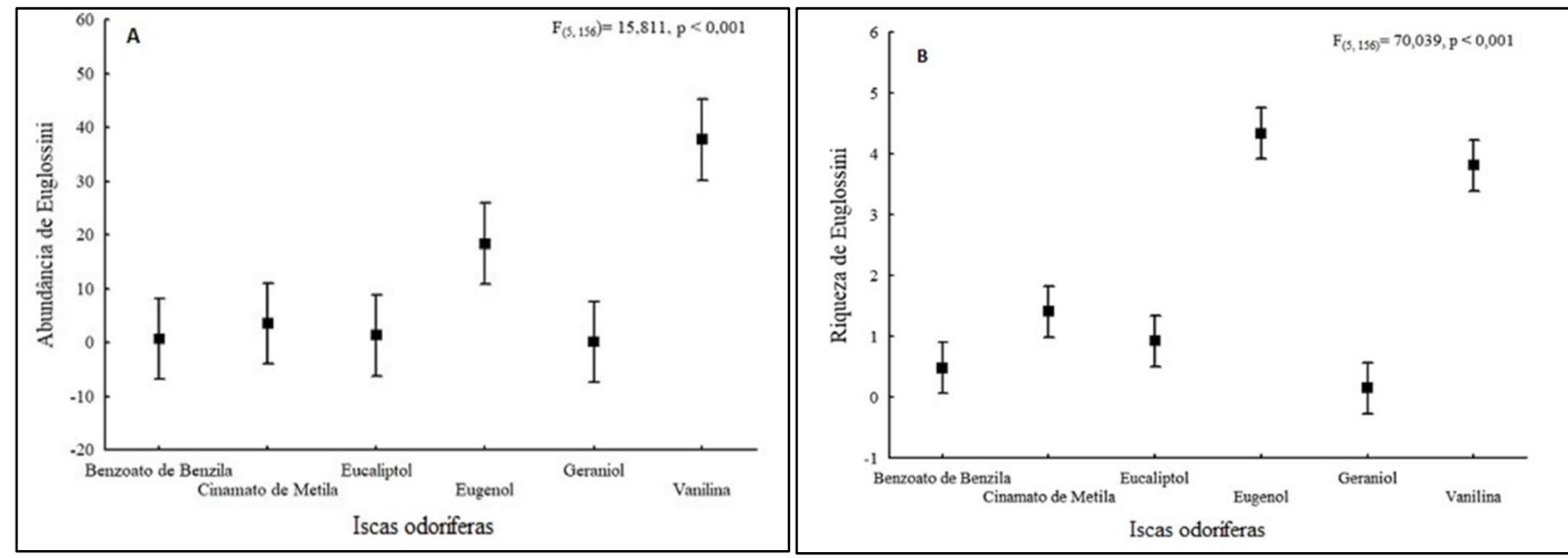

Figura 6: Comparação da (A) abundância e (B) riqueza de Euglossini na preferência pelas iscas odoríferas nas áreas de estudo no município de Mojuí dos Campos, Pará, Brasil.

Também houve diferença significativa da riqueza de espécies de Euglossini entre as iscas odoríferas $\left(F_{(5,156)}=70,039 ; p<0,001\right)$ (Figura $\left.6 \mathrm{~B}\right)$. O teste Tukey mostrou que eugenol foi a isca que atraiu o maior número de espécies, atraindo em média 1 espécie a mais que vanilina, 4 espécies a mais do que eucaliptol, geraniol e benzoato de benzila e 3 espécies a mais que cinamato de metila. Vanilina foi a segunda isca mais atrativa, atraindo 3 espécies a mais do que geraniol, eucaliptol e benzoato de benzila, e 2 espécies a mais do que cinamato de metila. As iscas geraniol, eucaliptol, benzoato de benzila e cinamato de metila não apresentaram diferença significativa em termos de riqueza.

\section{Abundância e riqueza de espécies entre os transectos dos fragmentos}

Não há diferença significativa na abundância $\left(F_{(2,24)}=1,851 p=0,178\right)$ e riqueza de espécies de Euglossini $\left(\mathrm{F}_{(2,24)}=0,299 ; p=0,744\right)$ entre os transectos borda, intermediário e centro das áreas estudadas. 


\section{DISCUSSÃO}

\section{Abundância e riqueza de espécies em distintos fragmentos}

A diferença na abundância de Euglossini entre os ambientes estudados está relacionada a quantidade de E. nigrita coletadas em grande número nos ambientes abertos de plantação de soja e pastagem, ambientes bem maiores que fragmentos de floresta. Esta espécie é típica de ambientes mais abertos e relativamente secos (REBÊLO et al., 1999), muito comum no cerrado (NEMÉSIO et al., 2004) e pantanal (FERREIRA et al., 2007), é uma espécie muito resistente a condições de estresse ambiental devido sua plasticidade, o que a torna um bioindicador de impactos ambientais, pois é muito comum em áreas perturbadas (CARVALHO FILHO, 2010).

No sul do Brasil, um estudo realizado em ambientes onde áreas de floresta nativas foram substituídas por pastagem, foi observado grande número de E. nigrita (SOFIA et al., 2004). No Acre, próximo à capital Rio Branco, em um estudo de levantamento da fauna de Euglossini, a mesma espécie foi uma das mais abundantes (STORCK-TONON et al., 2009). Em outro estudo de levantamento da fauna de Euglossini feito em área de transição entre Floresta Amazônica e Cerrado, Oliveira Junior et al. (2015), também observaram grande número de $E$. nigrita. A abundância dessa espécie também tem se mostrado significativa em muitos estudos realizados no Sul, Sudeste e Nordeste (BEZERRA et al., 2001; NEVES et al., 1997; NEMÉSIO, 2003; REBÊLO et al., 1997; PERUQUETTI et al., 1999; SOUZA et al., 2005).

Segundo Peruquetti et al. (1999) é esperado que fragmentos florestais maiores e menos perturbados possam oferecer mais recursos florais aos visitantes e, portanto, maior riqueza de espécies de abelhas. Essa hipótese pode ser uma possível explicação para não haver diferença significativa na comparação de riqueza entre as áreas estudadas, nesse sentido, devido os fragmentos de florestas serem consideravelmente menores que as áreas abertas de pastagem e plantação de soja em muitos locais, podem oferecer poucos recursos. Outro fator que pode influenciar nesse resultado, é a capacidade de dispersão dessas abelhas, podendo explorar localidades próximas (TONHASCA JUNIOR et al., 2002; 2003; GIEHL et al., 2013).

Pesquisas comparando riqueza de espécies de abelhas Euglossini entre fragmentos de floresta e áreas abertas são escassas, principalmente na Amazônia. No sul do Brasil, Sofia et al. (2004) realizaram um estudo semelhante e compararam a riqueza de machos Euglossini entre três fragmentos de florestas e não perceberam relação entre riqueza e tamanho dos fragmentos, concluindo que essas abelhas podem explorar localidades próximas em busca de recursos.

\section{Preferência por iscas odoríferas}

De forma geral, vanilina e eugenol têm se mostrado bastante atrativas em diversos estudos (PERUQUETTI et al., 1999; OLIVEIRA JUNIOR et al., 2015; SANTOS JÚNIOR et al., 2014). No presente trabalho, vanilina foi a isca odorífera com mais preferência, atraindo maior número em abundância de indivíduos, seguida por eugenol que atraiu maior número em riqueza de espécies, e a menos atrativa foi geraniol.

A preferência por essas iscas tem sido similar em alguns estudos e diferente em outros, por exemplo, 
Peruquetti et al. (1999) obteve resultado semelhante onde as iscas com eugenol atraíram maior número de espécie e vanilina maior número de indivíduo. Por outro lado, Oliveira Junior et al. (2015) observou que não houve diferença significativa entre essas duas iscas, porém, vanilina foi a isca mais atrativa para $E$. surinamensis. Ferreira et al. (2007), ao analisar a preferência por espécie, a vanilina mostrou-se o único composto atrativo para a espécie de Eufriesea cfr. auriceps. No entanto, no estudo de Giehl et al. (2013) vanilina mostrou-se pouco atrativa, atraindo um indivíduo apenas.

Alguns estudos também obtiveram resultados semelhantes em relação à maior número de espécies atraídas pela isca eugenol e espécimes por vanilina, ou vice-versa. Santos Júnior et al. (2014), também observaram resultado semelhante, onde eugenol atraiu maior número de espécies. Ferreira et al. (2011), observou em seu estudo resultado oposto, onde as armadilhas com eugenol coletaram maior número de espécimes, enquanto vanilina atraiu maior número de espécies.

Ao analisar tais estudos onde eugenol e vanilina mostraram diferencial na atração pelas abelhas, é possível observar que não há um padrão na preferência pelas iscas odoríferas. Compostos podem ser atrativos em uma região de estudo e em outras podem não ter efeito significativo. No caso da área estudada no município de Mojuí dos Campos, vários fatores podem ter influenciado para que houvesse abundância e riqueza significativa na preferência por vanilina e por eugenol, por exemplo, uma substância pode ser mais volátil que a outra, tendo uma melhor dispersão podendo atrair indivíduos de longas distâncias (SILVA et al., 2002). Além disso, fatores climáticos, a pureza e composição isométrica das substâncias (WILLIAMS et al., 1983), a estrutura etária das populações (ZIMMERMAN et al., 1988; REBÊLO et al., 1997), e a oferta das substâncias por fontes naturais também podem interferir na atividade das espécies.

A variação nas fragrâncias que são utilizadas pode concorrer para resultados indefinidos quanto às espécies que realmente são mais abundantes. Portanto, quanto mais estudos forem feitos, comparando poder de atratividade das fragrâncias na área estudada, assim como nas diferentes regiões do Brasil, mais eficazes serão os levantamentos faunísticos de Euglossini e maior será o entendimento da relação dessas abelhas com as substâncias aromáticas.

\section{Abundância e riqueza de espécies entre os transectos dos fragmentos}

Uma hipótese mais provável para explicar o fato de não haver significância tanto na riqueza de espécies quanto na abundância das abelhas Euglossini entre os transectos borda, intermediário e centro, está relacionada com a proximidade entre as áreas. Quando se analisa a ocorrência dessas abelhas, sempre espera-se obter maior abundância no interior da floresta, pelo fato de haver mais recursos, por exemplo, Morato (1994) observou maior abundância no interior da floresta do que na borda do fragmento da área estudada. Porém, a proximidade entre as áreas e a capacidade dessas abelhas de voar longas distâncias (JAZEN, 1971; KROODSMA, 1975), podem influenciar no resultado. Oliveira Junior et al. (2015), analisou o efeito de borda em três pontos de uma área, e em apenas um, observou diferença de riqueza entre espécie. Brosi et al. (2008), argumentam que a fragmentação florestal afeta de forma severa as abelhas Euglossini, o que pode reduzir a abundância na borda de florestas próximas a pastagem. 


\section{CONCLUSÕES}

De forma geral, as três áreas amostradas de Mojuí dos Campos apresentam alto grau de degradação e ação antrópica devido aos diferentes usos do solo. A abundância de E. nigrita capturadas parece mostrar um maior grau de perturbação no ambiente, reforçando a hipótese de que é uma espécie bioindicadora de alteração ambiental. Não foi observada diferença significativa na abundância no transecto centro em comparação com a borda e intermediário, provavelmente por fatores como tamanho dos fragmentos e proximidades entre eles. Em relação às iscas odoríferas, vanilina e eugenol foram as mais atrativas, onde vanilina atraiu maior número em abundância e eugenol maior número de riqueza de espécies. No entanto, são necessários outros estudos que possam comparar a riqueza de espécies entre um número maior de fragmentos, que levem em conta o tamanho da área, o grau de alteração ambiental, o número de machos de E. nigrita capturados, em relação a outras espécies, para que se possa obter dados mais precisos sobre os impactos dos diferentes usos do terra na diversidade dessas abelhas e outras espécies, e a importância de se preservar esses fragmentos, extremamente vitais para a preservação das abelhas Euglossini e a manutenção da polinização, serviço prestado por estes organismos a muitas espécies vegetais.

\section{REFERÊNCIAS}

ANJOS-SILVA, E. J. D.; REBÊLO, J. M. M.. A new species of Exaerete Hoffmannsegg (Hymenoptera: Apidae: Euglossini) from Brazil. Zootaxa, v.1105, n.1, p.27-35, 2006.

AYRES, M.; et al. BioEstat 5.0: aplicações estatísticas nas áreas das ciências biológicas e médicas. 2007.

BEZERRA, C. P.; MARTINS, C. F.. Diversidade de Euglossinae (Hymenoptera, Apidae) em dois fragmentos de Mata Atlântica localizados na região urbana de João Pessoa, Paraíba, Brasil. Revista Brasileira de Zoologia, v.18, n.3, p.823-835, 2001.

BEZERRA, E. D. S.; MACHADO, I. C.. Biologia floral e sistema de polinização de Solanum stramonifolium Jacq. (Solanaceae) em remanescente de Mata Atlântica, Pernambuco. Acta Botânica Brasílica, v.17, n.2, p.247-257, 2003.

BROSI, B. J.; DAILY, G. C.; SHIH, T. M.; OVIEDO, F.; DURÁN, G.. The effects of forest fragmentation on bee communities in tropical countryside. Journal of Applied Ecology, v.45, n.3, p.773-783, 2008.

CAMERON, S. A.. Phylogeny and biology of Neotropical orchid bees (Euglossini). Annual Reviews in Entomology, v.49, n.1, p.377-404, 2004.

CARVALHO, R.; MACHADO, I. C.. Pollination of Catasetum macrocarpum (Orchidaceae) by Eulaema bombiformis (Euglossini). Lindleyana, v.17, n.2, p.85-90, 2002.

CARVALHO FILHO, F. D. S.. Scent-robbing and fighting among male orchid bees, Eulaema (Apeulaema) nigrita Lepeletier, 1841 (Hymenoptera: Apidae: Euglossini). Biota Neotropica, v.10, n.2, p.405-408, 2010.

DEFRIES, R. S.; HOUGHTON, R. A.; HANSEN, M. C.; FIELD, C.
B.; SKOLE, D.; TOWNSHEND, J.. Carbon emissions from tropical deforestation and regrowth based on satellite observations for the 1980s and 1990s. Proceedings of the National Academy of Sciences, v.99, n.22, p.14256-14261, 2002.

DODSON, C. H.; DRESSLER, R. L.; HILLS, H. G.; ADAMS R. M.; WILLIAMS, N. H.. Biologically active compounds in orchid fragrances. Science, v.164, n.3885, p.1243-1249, 1969.

DRESSLER, R. L.. Pollination by euglossine bees. Evolution, v.22, n.1, p.202-210, 1968.

DRESSLER, R. L.. Biology of the orchid bees (Euglossini). Annual Review of Ecology and Systematics, v.13, n.1, p.373394, 1982.

FARIAS, R. C. A. P.; MARTINS, C. F.. Sazonalidade e padrões diários de atividade de machos de Euglossina (Hymenoptera: Apidae: Apini) e preferências por fragrâncias artificiais em um remanescente de Brejo de Altitude na Paraíba. EntomoBrasilis, v.6, n.3, p.202-209, 2013.

FERREIRA, P. E.; FREITAS, R. F.; AUGUSTO, S. C.. Diversidade de Euglossini (Hymenoptera: Apidae) em áreas de cerrado do triângulo mineiro, MG. Bioscience Journal, v.23, 2007.

FERREIRA, M. G.; PINHO, O. C.; BALESTIERI, J. B. P.; FACCENDA, O.. Fauna and stratification of male orchid bees (Hymenoptera: Apidae) and their preference for odor baits in a forest fragment. Neotropical Entomology, v.40, n.6, p.639-646, 2011.

GIEHL, N. F.; VALADÃO, M. B. X.; BRASIL, L. S.; DOS SANTOS, J. O.; ALMEIDA, S. M.; ENZA, L.; ANJO-SILVA, E. J.. O Efeito do Fogo sobre a Comunidade de Abelhas Euglossini (Hymenoptera: Apidae) em Floresta de Transição CerradoAmazônia (Mato Grosso, Brasil). EntomoBrasilis, v.6, n.3, 
p.178-183, 2013.

HILLS, H. G.; WILLIAMS, N. H.; DODSON, C. H.. Floral fragrances and isolating mechanisms in the genus Catasetum (Orchidaceae). Biotropica, v.4, n.2, p.61-76, 1972.

IBGE. Instituto Brasileiro de Geografia e Estatística. Bases e referenciais: bases cartográficas: malhas digitais. Rio de Janeiro: IBGE, 2015.

JAZEN, D. H.. Euglossine bees as long-distance pollinators of tropical plants. Science, v.171, n.3967, p.203-205, 1971.

KIMSEY, L. S.. Systematics of bees of the genus Eufriesea (Hymenoptera, Apidae). Univ of California Press, 1982.

KROODSMA, D. E.. Flight distances of male euglossine bees in orchid pollination. Biotropica, v.7, n.1, p.71-72, 1975.

LASALLE, J.; GLAULD, I. D.. Hymenoptera: their biodiversity, and their impact on the diversity of other organisms. Hymenoptera and Biodiversity, 1993.

MARTINI, P.; SCHLINDWEIN, C.; MONTENEGRO, A.. Pollination, flower longevity, and reproductive biology of Gongora quinquenervis Ruíz and Pavón (Orchidaceae) in an Atlantic forest fragment of Pernambuco, Brazil. Plant Biology, v.5, n.5, p.495- 503, 2003.

MICHENER, C. D.. The bees of the world. JHU press, 2000.

MINCKLEY, R. L.; REYES, S. G.. Capture of the orchid bee, Eulaema polychroma (Friese) (Apidae: Euglossini) in Arizona, with notes on northern distributions of other Mesoamerican bees. Journal of the Kansas Entomological Society, p.102104, 1996

MORATO, E. F.. Abundância e riqueza de machos de Euglossini (Hymenoptera: Apidae) em mata de terra firme e áreas de derrubada, nas vizinhanças de Manaus (Brasil). Boletim do Museu Paraense Emílio Goeldi, v.10, n.1, p.95105, 1994.

MOURE, J. S.. As espécies do gênero Eulaema Lepeletier, 1841 (Hymenoptera, Apidae, Euglossinae). Acta Biologica Paranaense, v. 29, 2000.

NEMÉSIO, A.. Preliminary sampling of Euglossina (Hymenoptera: Apidae: Apini) of Reserva Particular do Patrimônio Natural Feliciano Miguel Abdala, Caratinga, Minas Gerais, southeastern Brazil. Lundiana, v.4, n.2, p.121124, 2003

NEMÉSIO, A.; FARIA JUNIOR, L. R. R.. First assessment of the orchid-bee fauna (Hymenoptera: Apidae) at Parque Estadual do Rio Preto, a cerrado area in southeastern Brazil. Lundiana, v.5, n.2, p.113-117, 2004.

NEMÉSIO, A.; SILVEIRA, F. A.. Orchid bee fauna (Hymenoptera: Apidae: Euglossina) of Atlantic Forest fragments inside an urban area in southeastern Brazil. Neotropical Entomology, v.36, n.2, p.186-191, 2007.

NEMÉSIO, A.; SILVEIRA, F. A.. Forest fragments with larger core areas better sustain diverse orchid bee faunas (Hymenoptera: Apidae: Euglossina). Neotropical Entomology, v.39, n.4, p.555-561, 2010.
NEVES, E. D.; VIANA, B. F.. Inventário da fauna de Euglossinae (Hymenoptera, Apidae) do baixo sul da Bahia, Brasil. Revista Brasileira de Zoologia, v.14, n.4, p.831-837, 1997.

NEVES, E. L.; VIANA B. F.. Comunidade de machos de Euglossinae (Hymenoptera, Apidae) das matas ciliares da margem esquerda do médio Rio São Francisco, Bahia. Anais da Sociedade Entomológica do Brasil, v.28, n.2, p.201-210, 1999.

OLIVEIRA, M. L.. Três novas espécies de abelhas da Amazônia pertencentes ao gênero Eulaema (Hymenoptera: Apidae: Euglossini). Acta Amazônica, v.36, n.1, p.121-128, 2006.

OLIVEIRA JUNIOR, J. M. B.; ALMEIDA, S. M.; RODRIGUES, L.; SILVERIO JUNIOR, A. J.; ANJOS-SILVA, E. J.. Orchid bees (Apidae: Euglossini) in a forest fragment in the ecotone Cerrado-Amazonian forest, Brazil. Acta Biológica Colombiana, v 20, n.3, 2015.

PERUQUETTI, R. C.; CAMPOS, L. A. O.; COELHO, C. D. P.; ABRANTES, C. V. M.; LISBOA, L. C. O.. Abelhas Euglossini (Apidae) de áreas de Mata Atlântica: abundância, riqueza e aspectos biológicos. Revista Brasileira de Zoologia, v.16, n.2, p.101-118, 1999.

POWELL, A. H.; POWELL G. N. N.. Population dynamics of male euglossine bees in Amazonian forest fragments. Biotropica, v.19, n.2, p.176-179, 1987.

R DEVELOPMENT CORE TEAM. R: A language and environment for statistical computing. Vienna: R Foundation for Statistical Computing, 2011.

RAMALHO A. V.; GAGLIANONE, M. C.; OLIVEIRA, M. L.. Comunidades de abelhas Euglossina (Hymenoptera, Apidae) em fragmentos de Mata Atlântica no sudeste do Brasil. Revista Brasileira de Entomologia, v.53, n.1, p.95-101, 2009.

REBÊLO, J. M. M.; GARÓFALO, C. A.. Comunidades de machos de Euglossini (Hymenoptera: Apidae) em matas semidecíduas do nordeste do estado de São Paulo. Anais da Sociedade Entomológica do Brasil, v.26, n.2, p.243-255, 1997.

REBÊLO, J. M. M.; SILVA F. S.. Distribuição das abelhas Euglossini (Hymenoptera: Apidae) no Estado do Maranhão, Brasil. Anais da Sociedade Entomológica do Brasil, v.28, n.3, p.389-401, 1999.

REBÊLO, J. M. M.. História Natural das Euglossíneas. As abelhas das orquídeas. São Luís: Lithograf, 2001.

ROUBIK, D. W.; HANSON, P. E.. Orchid bees of tropical America: biology and field guide. Heredia: Instituto Nacional de Biodiversidad (INBio), 2004.

ROUBIK, D. W.. Long-term studies of solitary bees: what the orchid bees are telling us. Solitary bees, 2004.

SANTOS JÚNIOR, J. S.; FERRARI, R. R.; NEMÉSIO, A.. The orchid-bee fauna (Hymenoptera: Apidae) of a forest remnant in the southern portion of the Brazilian Amazon. Brazilian Journal of Biology, v.74, n.3, p.184, 2014. 
SILVA, F. S.; REBÊLO, J. M. M.. Population dynamics of Euglossinl bees (Hymenoptera, Apidae) in an early secondgrowth forest of Cajual Island, in the state of Maranhão, Brazil. Brazilian Journal of Biology, v.62, n.1, p.15-23, 2002.

SOFIA, S. G.; SUZUKI, K. M.. Comunidades de machos de abelhas Euglossina (Hymenoptera: Apidae) em fragmentos florestais no sul do Brasil. Neotropical Entomology, v.33, n.6, p.693-702, 2004.

SOUZA, A. P. K.; HERNÁNDEZ M. I. M.; MARTINS, C. F.. Riqueza, abundância e diversidade de Euglossina (Hymenoptera, Apidae) em três áreas da Reserva Biológica Guaribas, Paraíba, Brasil. Revista Brasileira de Zoologia, v.22, n.2, p.320-325, 2005.

STORCK-TONON, D.; MORATO, E. F.; OLIVEIRA, M. L.. Fauna de Euglossina (Hymenoptera: Apidae) da Amazônia SulOcidental, Acre, Brasil. Acta Amazônica, v.39, n.3, p.693 706, 2009.

TONHASCA JUNIOR, A.; BLACKMER, J. L.; ALBUQUERQUE, G. S.. Abundance and Diversity of Euglossine Bees in the Fragmented Landscape of the Brazilian Atlantic Forest.
Biotropica, v.34, n.3, p.416-422, 2002.

TONHASCA JUNIOR, A.; ALBUQUERQUE, G. S.; BLACKMER, J. L.. Dispersal of euglossine bees between fragments of the Brazilian Atlantic Forest. Journal of Tropical Ecology, v.19, n.1, p.99-102, 2003.

WILLIAMS, N. H.; WHITTEN, W. M.. Orchid floral fragrances and male euglossine bees: methods and advances in the last sesquidecade. The Biological Bulletin, v.164, n.3, p.355-395, 1983.

ZAR, J. H.. Biostatistical analysis. 5 ed. Upper Saddle River, 2010.

ZANELLA, F. C.. The bees of the Caatinga (Hymenoptera, Apoidea, Apiformes): a species list and comparative notes regarding their distribution. Apidologie, v.31, n.5, p.579-592, 2000.

ZIMMERMAN, J. K.; MADRIÑÁN, S.. Age structure of male Euglossa imperialis (Hymenoptera: Apidae: Euglossini) at nectar and chemical sources in Panama. Journal of Tropical Ecology, v.4, n.3, p.303-306, 1988.

A CBPC - Companhia Brasileira de Produção Científica (CNPJ: 11.221.422/0001-03) detém os direitos materiais desta publicação. Os direitos referem-se à publicação do trabalho em qualquer parte do mundo, incluindo os direitos às renovações, expansões e disseminações da contribuição, bem como outros direitos subsidiários. Todos os trabalhos publicados eletronicamente poderão posteriormente ser publicados em coletâneas impressas sob coordenação da Sustenere Publishing, da Companhia Brasileira de Produção Científica e seus parceiros autorizados. Os (as) autores (as) preservam os direitos autorais, mas não têm permissão para a publicação da contribuição em outro meio, impresso ou digital, em português ou em tradução. 